\title{
Drug Resistance and Serotyping of Uropathogenic Escherichia coli Among Patients With Urinary Tract Infection in Rasht, Iran
}

\author{
Khosro Issazadeh ${ }^{1, *}$; Seyedeh Nasrin Naghibi ${ }^{1}$; Mohammad Reza Majid Khoshkholgh- \\ Pahlaviani $^{2}$ \\ ${ }^{1}$ Department of Microbiology, Faculty of Basic Sciences, Lahijan Branch, Islamic Azad University, Lahijan, IR Iran \\ ${ }^{2}$ Department of Biotechnology, Faculty of Basic Sciences, Lahijan Branch, Islamic Azad University, Lahijan, IR Iran \\ *Corresponding author: Khosro Issazadeh, Department of Microbiology, Faculty of Basic Sciences, Lahijan Branch, Islamic Azad University, Lahijan, IR Iran. \\ E-mail: issa_kaam@yahoo.com
}

Received: April 9, 2014; Accepted: June 5, 2014

\begin{abstract}
Background: Urinary Tract Infection (UTI) is one of the most common reasons for human to seek medical advice and is also one of the most frequently occurring nosocomial infections. Nosocomial UTIs are almost exclusively complicated with a broad spectrum of etiological pathogens. Escherichia coli remains the predominant uropathogen (80\%) isolated in community-acquired infections.

Objectives: The aim of this study was to determine the local incidence of UTIs, identification of Uropathogenic E. coli (UPEC), serotyping and strains antimicrobial resistance of $E$. coli isolated from these patients.

Patients and Methods: In this prospective cross-sectional study, in order to isolate and identify bacterial agent (UPEC) causing UTI and to identify it's sex and age distribution, a total 150 urine samples through simple random sampling method were analyzed for identification of bacterial isolates. Lactose fermenting colonies on MacConkey's agar were processed and identified as E. coli by standard biochemical tests. Antibiotic susceptibility tests were performed with disk diffusion tests using Kirby-Bauer method on E. coli isolated from 110 cases with UTI aged from 1 year to 85 years and serotyping achieved by slide agglutination method. All data analyzed using SPSS-21 software.

Results: Majority of UPEC belonged to serotype O1, O2, O4, O20, O25, O44, 064 and O78. Majority of the isolates were resistant to routinely used antibiotics amoxicillin, cotrimoxazole, tetracycline and gentamicin. Most of the isolates were sensitive to nalidixic acid, ciprofloxacin,
\end{abstract} nitrofurantoin and ceftazidime.

Conclusions: The present study revealed that serotype $\mathrm{O} 25$ was isolated with maximum frequency followed by $\mathrm{O} 2, \mathrm{O} 6, \mathrm{O} 1, \mathrm{O} 78, \mathrm{O} 44, \mathrm{O} 20$ and 064 respectively.

Keywords: Urinary tract infection; Uropathogenic Escherichia coli; Antimicrobial susceptibility

\section{Background}

Urinary tract infections are one of the most common types of bacterial infections in humans occurring both in the community and the health care settings and ranks high amongst the most common reasons that compel an individual to seek medical attention $[1,2]$. Urinary tract infection is emerging as an important community acquired and nosocomial bacterial infection. Moreover, antimicrobial resistance to various classes of antimicrobials continues to be major health problem in different parts of the world [3-6].

Escherichia coli is the most common pathogen associated with Urinary Tract Infection (UTI) [7]. E. coli that are associated with UTI are commonly named uropathogenic isolates, although there is evidence that different pathotypes may be related to UTI [8]. Antimicrobial resistance among UPEC shows considerable variations during different time periods and in different areas. The problem becomes further complicated with the emergence of extended-spectrum beta-lactamase (ESBL) producing UPEC
$[9,10]$. Fifty percent of all women will experience at least one time UTI in their lifetime and, of those, about $25 \%$ will have one or more recurrent infections [11]. The structure of the females urethra and vagina makes it susceptible to trauma during sexual intercourse as well as bacteria been massaged up the urethra and into the bladder during pregnancy and or child birth $[1,2]$. Host factors such as the epithelial cell receptivity are also important in the infection process. Although fungi and viruses are occasional etiological agents, UTIs are predominantly caused by bacteria. The most common bacteria implicated as causative agents of UTI generally originate in the intestine and the major etiological agent, Uropathogenic $E$. coli (UPEC), is responsible for approximately $90 \%$ of all UTIs [12].

Extremes of age, female gender, pregnancy, instrumentation, UTI, neurologic dysfunction, renal disease and expression of $\mathrm{A}, \mathrm{B}$, and $\mathrm{H}$ blood groups oligosaccharides on the surface of epithelial cells are predisposing factors 
for the development of UTIs [1]. E. coli strains are normally identified by serological typing of their $\mathrm{H}$ (flagellar), $\mathrm{O}$ (lipopolysaccharide) and in some cases, $\mathrm{K}$ (capsular) surface antigens. Since, 176 O-serogroups had been described for E. coli [12]. In UPEC, the O-serogroups is related to the virulence factor profile of each strain. Previous studies reported that $\mathrm{O} 1, \mathrm{O} 2, \mathrm{O} 4, \mathrm{O} 6, \mathrm{O} 7, \mathrm{O} 8, \mathrm{O} 15, \mathrm{O} 16, \mathrm{O} 18, \mathrm{O} 21, \mathrm{O} 22$, O25, $\mathrm{O} 75$ and 083 sero-groups are preferentially associated with UPEC strains $[13,14]$. Treatment of UTIs caused by this bacterium often requires antimicrobial therapy. Several studies showed that antibiotic resistance in UPEC is increasing in these days $[15,16]$.

\section{Objectives}

Despite the high prevalence of UTI very few studies have been conducted to determine the prevalence of various serotypes of $E$. coli this study was carried out for determination of sero-groups, and antimicrobial resistance properties of UPEC strains isolated from patients with UTIs in Iran.

\section{Patients and Methods}

This prospective cross-sectional study was conducted in the Department of Microbiology, Islamic Azad University of Lahijan, between January 2012 and January 2013. Urine samples were collected from patients admitted to Razi, 17 Shahrivar and Poursina hospitals in Rasht (North of Iran). Cases showing pyuria and significant bacterial growth on culture were included in the study. Women with symptoms of uncomplicated lower UTI were eligible for inclusion. Briefly, besides women with uncomplicated cystitis, patients with recurrent UTI ( $\geq 3$ episodes in the last year) as well as pregnant and diabetic women were included. Patients with upper UTI, concomitant or prophylactic antimicrobial treatment within 15 days before inclusion in the study, history of renal impairment, and anatomical and functional urinary tract abnormalities were excluded. These patients did not include those who were on antibiotics a week before the samples were collected.

A total of 110 E. coli strains were isolated with UTIs symptoms (70 females and 40 males). In this study, age groups of patients were between 1 - 85 years old. Bacterial count was more than $10^{5} / \mathrm{mL}$ in freshly voided midstream urine samples. These samples were processed on blood agar and MacConkey medium (Merck Co. LTD. Germany) with a standard loop and were incubated at $37^{\circ} \mathrm{C}$ overnight and observed for growth through formation of colonies. All isolated bacteria and lactose fermenting colonies on MacConkey's Agar (MA) showing significant bacteriuria were processed and identified as E. coli by standard biochemical tests.

\subsection{Serotyping}

Slide agglutination was performed according to the manufacturer's guidelines for the Kit (Mast Co., UK and Sifin Co., Germany). At the beginning, heat treated suspension of the organism was prepared and labeled as an- tigen suspension, one loop full of this suspension were placed on a microscopic slide. One drop of antiserum was placed on one drop of suspension and one drop of normal saline was placed on the other, which served as control. The regents were mixed by titling back and forth for 60 seconds while viewing it under indirect light against a dark background.

Distinct clumping or agglutination within this period, along with absence of clumping in the saline control, was regarded as positive result.

\subsection{Antibiotic Susceptibility Testing}

The antibacterial susceptibility testing of the isolates was done using the Kirby-Bauer disk diffusion method. According to the definition of the Clinical and Laboratory Standards Institute (CLSI) using antibiotics containing discs from Oxoid. Briefly, $20 \mathrm{~mL}$ of Mueller-Hinton agar (Merck Co. Ltd, Germany) was prepared and poured into sterile plates.

The agar medium was allowed to solidify at room temperature on a flat bench. Then some few colonies of an 18 hours culture of the isolates were streaked on the surfaces of the well-dried agar plates. Then some antibiotic discs were gently and firmly placed on the agar plates, which were then left at room temperature for $1 \mathrm{~h}$ to allow diffusion of the antibiotics into the agar medium. The plates were then incubated at $35-37^{\circ} \mathrm{C}$ for $24 \mathrm{~h}$. Zones of growth inhibition were then measured to the nearest millimeter and recorded. The mean of triplicate results was taken as the zone diameter. The antibiotics discs used were ciprofloxacin, nitrofurantoin, amoxicillin, cotrimoxazole, tetracycline, gentamicin, nalidixic acid, and ceftazidime. Isolates were classified as either resistant or intermediate sensitive or sensitive based on the definition of the Clinical and Laboratory Standard Institute (CLSI, 2006) and accordance with WHO requirements [17]. E. coli ATCC25922 was used for quality control purposes.

Statistical analysis was performed using SPSS-21 software. Descriptive analyses were done for parametric and nonparametric variables. $\chi^{2}$ test was used for evaluation of correlation between antibiotic resistant patterns, and clinical diagnosis with serotype. Relationship between age group and gender of the patients and serotype was analyzed by t-test. P-Value $<0.05$ were considered as statistically significant.

\section{Results}

Totally, 110 strains of E. coli were isolated from urine samples with UTI, and aged one year to 85 years. The female to male ratio was 1.75 , with $63.6 \%$ of the patients being females, uncomplicated UTI was diagnosed in $49 \%$, pyelonephritis in $17.3 \%$, cystitis in $16.4 \%$, and Pyrexia of Unknown Origin (PUO) in 9.1\% and prostitutes in $8.2 \%$ of these patients.

Serotyping of the 110 studied strains revealed that they belong to diverse serogroups. Eighty-two (74.5\%) were O- 
Issazadeh Ket al.

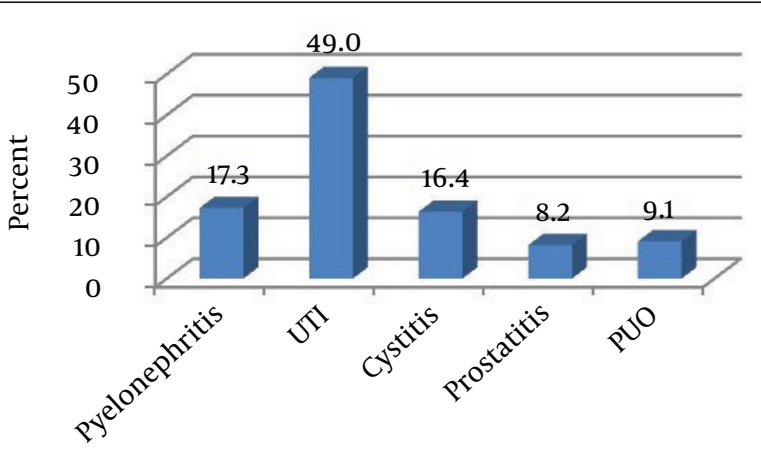

Clinical diagnosis

Figure 1. Clinical Manifestations of Patients With Urinary Tract Infection

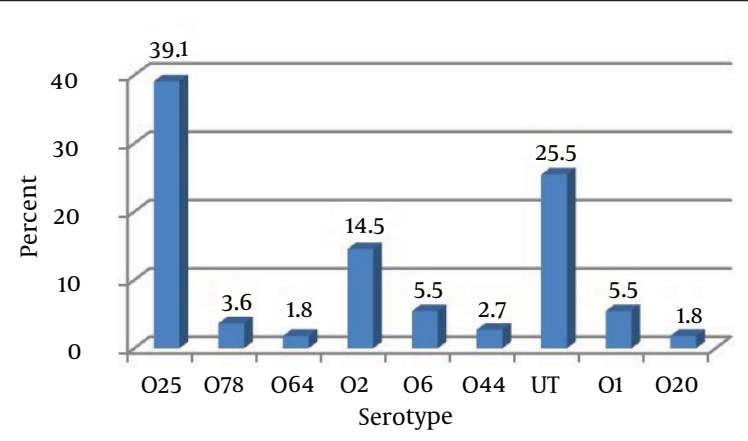

Figure 2. Serotypes of UPEC Strains Isolated From Patients With Urinary Tract Infection

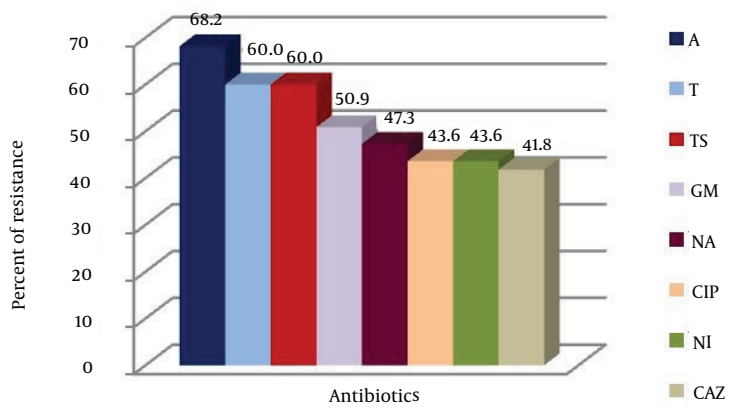

Figure 3. Antibiotic Resistance of UPEC Strains Isolated From Patients With Urinary Tract Infection

antigen typeable, 28 (25.5\%) were non-typeable because they did not react with any of the antisera used. Eighty two typeable strains belonged to serotypes $\mathrm{O} 1, \mathrm{O} 2, \mathrm{O}$, O20, O25, O44, 064 and O78. In total, $\mathrm{O} 25$ and $\mathrm{O} 2$ respectively with $39.1 \%$ and $14.5 \%$ had a highest and 020 , O64, O44, O78, O6 and 01 respectively with $1.8 \%, 1.8 \%, 2.7 \%, 3.6 \%$, $5.5 \%$ and $5.5 \%$ had a lowest prevalence of UPEC serogroups. The disk diffusion method in Mueller-Hinton agar showed that the UPEC serogroups had $68.2 \%, 60 \%, 60 \%$, $50.9 \%, 47.3 \%, 43.6 \%, 43.6 \%$ and $41.8 \%$ resistance to amoxicillin, cotrimoxazole, tetracycline, gentamicin, nalidixic acid, ciprofloxacin, nitrofurantoin and ceftazidime respectively. Highest resistance to amoxicillin (68.2\%) was and lowest resistance to ceftazidime (41.8\%) (Figure 1). In this study, the differences between gender, age groups and frequency of UPEC were statistically significant $(\mathrm{P}=$ 0.01). Also, the differences between clinical manifestations, age groups and frequency of UPEC were statistically significant $(\mathrm{P}<0.05)$. There was statistically significant correlation between serotypes of $\operatorname{UPEC}(\mathrm{P}=0.01)$. The present study indicated that there was no correlation between antibiotic resistance of UPEC serotypes compared with age of patients with exception of ciprofloxacin, nitrofurantoin and nalidixic acid $(\mathrm{P}<0.05)$. There was statistically significant correlation between clinical manifestations and antibiotic resistance of ciprofloxacin, nitrofurantoin and ceftazidime $(\mathrm{P}<0.05)$.

\section{Discussion}

UTI was present in 110 (73.33\%) of our patients. A total of 110 clinical isolates, 70 (63.6\%) and 40 (36.4\%) of cases were females and males, respectively. Among these 110 cases, the most frequent of UPEC strains among patients were older than 60 years of age than for others and the lowest rate of frequency belonged to 41 - 50 age groups. In this investigation, it was indicated that uncomplicated UTI was the most frequents of clinical manifestations. The assessment of serotyping profile between UPEC strains serotype $\mathrm{O} 25$ was the highest value and serotypes $\mathrm{O} 20$ and $\mathrm{O} 64$ showed the lowest rate. In assessing the association between antibiotic susceptibility pattern indicated that high resistance rates to amoxicillin (68.2\%) and the rate of ceftazidime resistance was found to be low (68.2\% of the isolates).

Our results indicated that the UPEC can be a major causative agent for UTIs in humans in Iran and this finding was similar with previous study which mentioned that 150 million people are diagnosed with UTI annually. In addition to this, our study showed that resistance pattern of UPEC strains to antimicrobial drugs has increased significantly and this is similar to previous study [18]. In the present study, E. coli strains showed high resistance to amoxicillin $68.2 \%$, cotrimoxazole $60 \%$, tetracycline $60 \%$ and gentamicin $50.9 \%$ while being very susceptible to ceftazidime $58.2 \%$, nitrofurantoin $56.4 \%$, ciprofloxacin $56.4 \%$ and nalidixic acid $52.7 \%$.

These results show that UPEC strains in Iran have become sufficiently resistant to treatment with amoxicillin, fluoroquinolones and trimethoprim-sulfamethoxazole, as to render their use contraindicated. In the past, trimethoprim-sulfamethoxazole was used as the standard antibiotic for a calculated UTIs therapy and due to the increased resistance of UPEC strains to this class of antibiotics, fluoroquinolones as broad-spectrum 
antimicrobial agents have been used with increasing frequency in complicated as well as uncomplicated UTIs [19], but after a short time resistance to fluoroquinolones was emerged [20]. Previous study showed that more than $10 \%$ of the E. coli isolated in $2000-2002$ from inpatients in European and North American were resistant against ciprofloxacin [21], while this amount of resistance was increased significantly in our study. The reasons for this finding are not clear, but may be related to differences in rates of antibiotic exposure, comorbidities and/or differences in antibiotic metabolism across different patient populations.

We studied types of O-antigen in UTI isolated E. coli strains. Totally, 82 samples were positive for one type of $\mathrm{O}$ antigen, with the most common of them were $\mathrm{O} 25$ (39.1\%) and O2 (14.5\%). E. coli strains were classified based on various types of O-antigen for the first time by Kauffmann-White 176 types of O-antigen have been detected till now. Various studies have been conducted in other centers for serotyping of uropathogenic E. coli. One of the earliest studies was conducted by Korsakov and Birch-Andersen. Their study on $\mathrm{O}, \mathrm{K}$ and $\mathrm{H}$ serotype determination of $267 \mathrm{E}$. coli strains in patients with pyelonephritis or cystitis led them to consider some $\mathrm{O}$, K and $\mathrm{H}$ types, for example, O6: $\mathrm{K} 2$ : $\mathrm{H} 1$ as pyelonephritis associated types and O6: K13: $\mathrm{H} 1$ as cystitis associated types. In that study, $\mathrm{O} 6$ was commonly seen in patients with both pyelonephritis and cystitis [22]. In this study, there was no significant correlation between cystitis and/or pyelonephritis and the type of O-antigen. In a study by Blanco et al. most uropathogenic E. coli serogroups belonged to $\mathrm{O} 1, \mathrm{O} 2, \mathrm{O} 4, \mathrm{O} 6, \mathrm{O} 9, \mathrm{O} 18, \mathrm{O} 27, \mathrm{O} 33, \mathrm{O} 57$, and O77 [23]. It seems that strains belonging to these serogroups possess specific virulence factors which confer on their special invasive ability. Our results almost support this theory.

The most common type of $\mathrm{O}$ antigen was $\mathrm{O} 2, \mathrm{O} 4$ and O6 [23]. Serotype 0101 was found to be the commonest serotype of uropathogenic E. coli causing UTI in two studies from India [24, 25]. Vranes et al. examined 160 E. coli strains isolated from UTI 75.6\% belonged to one of the fifteen serogroups $\mathrm{O} 1, \mathrm{O} 2, \mathrm{O} 4, \mathrm{O} 5, \mathrm{O} 6, \mathrm{O} 7, \mathrm{O} 8, \mathrm{O} 9, \mathrm{O} 11$, $\mathrm{O} 15, \mathrm{O} 17, \mathrm{O} 18, \mathrm{O} 25, \mathrm{O} 50$ and $\mathrm{O} 75$ while $24.4 \%$ were O-nontypable. Predominant serotypes were $\mathrm{O} 2, \mathrm{O} 4$ and $\mathrm{O} 6$ [26]. In Mexico City by Molina-Lopez et al. 119 E. coli isolates were tested and serotypes belonged to $\mathrm{O} 1, \mathrm{O} 2, \mathrm{O}$, O18 and 075 [27]. In a study by Zhao et al. serotyping of the uropathogenic strains revealed that 124 (61\%) were O-antigen typeable and 78 (39\%) were O-antigen nontypable. O type $\mathrm{O} 1$ (35 isolates) was the most prevalent among the typeable strains [28]. Although serotype 06 was the most common type found in many studies. In our present study, $\mathrm{O} 25$ and $\mathrm{O} 2$ were the most common type. In the present study, no correlation was seen between type of $\mathrm{O}$ antigen and antibiotic resistance. This is the first report of E. coli serotyping in human with UTI from North of Iran.

\section{Acknowledgements}

The authors appreciated the financial supports of Research Deputy and management of Microbiology Department, and respected experts in order to successfully implement the project number 20230507911002 in Islamic Azad University; Lahijan branch.

\section{Authors' Contributions}

All authors had equal role in design, work, statistical analysis and manuscript writing.

\section{Funding/Support}

Lahijan Branch, Islamic Azad University.

\section{References}

1. Al Sweih N, Jamal W, Rotimi VO. Spectrum and antibiotic resistance of uropathogens isolated from hospital and community patients with urinary tract infections in two large hospitals in Kuwait. Med Princ Pract. 2005;14(6):401-7.

2. Kolawole AS, Kolawole OM, Kandaki-Olukemi YT, Babatunde SK, Durowade KA, Kolawole CF. Prevalence of urinary tract infections (UTI) among patients attending Dalhatu Araf Specialist Hospital, Lafia, Nasarawa State, Nigeria. Int $j$ med sci. 2009;1(5):163-7.

3. Foxman B. The epidemiology of urinary tract infection. Nat Rev Urol. 2010;7(12):653-60.

4. Oliveira FA, Paludo KS, Arend LN, Farah SM, Pedrosa FO, Souza EM, et al. Virulence characteristics and antimicrobial susceptibility of uropathogenic Escherichia coli strains. Genet Mol Res 2011;10(4):4114-25.

5. Vila J, Pal T. Update on antibacterial resistance in low-income countries: factors favoring the emergence of resistan. The Open Infect Dis J. 2010;4(2):38-54.

6. Farshad S, Ranjbar R, Japoni A, Hosseini M, Anvarinejad M, Mohammadzadegan R. Microbial susceptibility, virulence factors, and plasmid profiles of uropathogenic Escherichia coli strains isolated from children in Jahrom, Iran. Arch Iran Med. 2012;15(5):312-6.

7. Gyles CL, Fairbrother JM. Escherichia Coli. In: Gyles CL, Prescott JF, Songer G editors. Pathogenesis of bacterial infections in animals. $4^{\text {th }}$ ed. New Jersey: Wiley Blackwell; 2010. 267-308.

8. Marrs CF, Zhang L, Foxman B. Escherichia coli mediated urinary tract infections: are there distinct uropathogenic E. coli (UPEC) pathotypes? FEMS Microbiol Lett. 2005;252(2):183-90.

9. Azap OK, Arslan H, Serefhanoglu K, Colakoglu S, Erdogan $\mathrm{H}$ Timurkaynak F, et al. Risk factors for extended-spectrum betalactamase positivity in uropathogenic Escherichia coli isolated from community-acquired urinary tract infections. Clin Microbiol Infect. 2010;16(2):147-51.

10. DeBusscher J, Zhang L, Buxton M, Foxman B, Barbosa-Cesnik C. Persistent extended-spectrum beta-lactamase urinary tract infection. Emerg Infect Dis. 2009;15(11):1862-4.

11. Dhakal BK, Kulesus RR, Mulvey MA. Mechanisms and consequences of bladder cell invasion by uropathogenic Escherichia coli. Eur J Clin Invest. 2008;38 Suppl 2:2-11.

12. Wiles TJ, Kulesus RR, Mulvey MA. Origins and virulence mechanisms of uropathogenic Escherichia coli. Exp Mol Pathol. 2008;85(1):11-9.

13. Abe CM, Salvador FA, Falsetti IN, Vieira MA, Blanco J, Blanco JE, et al. Uropathogenic Escherichia coli (UPEC) strains may carry virulence properties of diarrhoeagenic E. coli. FEMS Immunol Med Microbiol. 2008;52(3):397-406.

14. Yamamoto S. Molecular epidemiology of uropathogenic Escherichia coli. J Infect Chemother. 2007;13(2):68-73. 
15. Johnson JR, Kuskowski MA, O'Bryan T T, Colodner R, Raz R. Virulence genotype and phylogenetic origin in relation to antibiotic resistance profile among Escherichia coli urine sample isolates from Israeli women with acute uncomplicated cystitis. Antimicrob Agents Chemother. 2005;49(1):26-31.

16. Johnson JR, Johnston B, Kuskowski MA, Colodner R, Raz R. Spontaneous conversion to quinolone and fluoroquinolone resistance among wild-type Escherichia coli isolates in relation to phylogenetic background and virulence genotype. Antimicrob Agents Chemother. 2005;49(11):4739-44.

17. Onanuga A, Oyi AR, Olayinka BO, Onaolapo JA. Prevalence of community-associated multi-resistant Staphylococcus aureus among healthy women in Abuja, Nigeria. African J Biotec. 2005;4(9):942-5.

18. von Baum H, Marre R. Antimicrobial resistance of Escherichia coli and therapeutic implications. Int J Med Microbiol. 2005;295(67):503-11.

19. Gupta K, Scholes D, Stamm WE. Increasing prevalence of antimicrobial resistance among uropathogens causing acute uncomplicated cystitis in women. JAMA. 1999;281(8):736-8.

20. Kern WV, Oethinger M, Jellen-Ritter AS, Levy SB. Non-target gene mutations in the development of fluoroquinolone resistance in Escherichia coli. Antimicrob Agents Chemother: 2000;44(4):814-20.

21. Jones ME, Draghi DC, Thornsberry C, Karlowsky JA, Sahm DF, Wenzel RP. Emerging resistance among bacterial pathogens in the intensive care unit--a European and North American Surveillance study (2000-2002). Ann Clin Microbiol Antimicrob. 2004;3:14.

22. Korsakov F, Birch-Andersen AO. Fibril antigens in E. coli serotypes associated with pyelonephritis and cystitis. Scand J Infect Dis Suppl. 1982;33:18-25.

23. Blanco M, Blanco J, Blanco JE, Alonso MP, Abalia I, Rodriguez E, et al. [Virulence factors and 0 serogroups of Escherichia coli as a cause of community-acquired urinary infections]. Spanish. Enferm Infecc Microbiol Clin. 1995;13(4):236-41.

24. Mandal P, Kapil A, Goswami K, Das B, Dwivedi SN. Uropathogenic Escherichia coli causing urinary tract infections. Indian J Med Res. 2001;114:207-11.

25. Srikanth NS, Macaden R. Uropathogenic Escherichia coli--a preliminary study. Indian J Pathol Microbiol. 2003;46(1):145-6.

26. Vranes J, Schonwald S, sterk-Kuzmanovic N, Ivancic B. Low virulence of Escherichia coli strains causing exacerbation of chronic pyelonephritis. Acta Clinica Croatica. 2001;40(3):165-70.

27. Molina-Lopez J, Aparicio-Ozores G, Ribas-Aparicio RM, GavilanesParra S, Chavez-Berrocal ME, Hernandez-Castro R, et al. Drug resistance, serotypes, and phylogenetic groups among uropathogenic Escherichia coli including O25-ST131 in Mexico City. J Infect Dev Ctries. 2011;5(12):840-9.

28. Zhao L, Chen X, Zhu X, Yang W, Dong L, Xu X, et al. Prevalence of virulence factors and antimicrobial resistance of uropathogenic Escherichia coli in Jiangsu province (China). Urology. 2009;74(3):702-7. 